\title{
A NONLINEAR ERGODIC THEOREM FOR AN AMENABLE SEMIGROUP OF NONEXPANSIVE MAPPINGS IN A HILBERT SPACE
}

\author{
WATARU TAKAHASHI
}

\begin{abstract}
We prove a nonlinear ergodic theorem for noncommutative semigroups of nonexpansive mappings in a Hilbert space. Furthermore, we give a necessary and sufficient condition for a noncommutative semigroup to have a fixed point.
\end{abstract}

1. Introduction. Let $\boldsymbol{H}$ be a real Hilbert space with norm $\|\cdot\|$ and inner product $\langle\cdot, \cdot\rangle$ and $C$ a nonempty closed convex subset of $H$. A mapping $T: C \rightarrow C$ is called nonexpansive on $C$, or $T \in \operatorname{Cont}(C)$ if

$$
\|T x-T y\|<\|x-y\| \text { for every } x, y \in C \text {. }
$$

Let $F(T)$ be the set of fixed points of $T$, that is, $F(T)=\{z \in C: T z=z\}$. Then, the set $F(T)$ is obviously closed and convex. Let $S=\{S(t): t>0\}$ be a family of nonexpansive mappings of $C$ into itself such that $S(0)=I, S(t+s)=S(t) S(s)$ for all $t, s \in[0, \infty)$ and $S(t) x$ is continuous in $t \in[0, \infty)$ for each $x \in C$. Then, $S$ is called a nonexpansive semigroup on $C$. The fixed point set $F(S)$ of $S$ is defined by

$$
F(S)=\{x \in C: S(t) x=x \text { for all } t \in[0, \infty)\} .
$$

The first nonlinear ergodic theorem for nonexpansive mappings was established by Baillon [1]: Let $C \subset H, T \in \operatorname{Cont}(C)$ and $F(T) \neq \varnothing$. Then the Cesàro means

$$
S_{n}(x)=\frac{1}{n} \sum_{k=0}^{n-1} T^{k} x
$$

converge weakly as $n \rightarrow+\infty$ to a fixed point of $T$ for each $x \in C$. A corresponding result for nonexpansive semigroups on $C$ was given by Baillon [2] and Baillon-Brézis [3]. Nonlinear ergodic theorems for general commutative semigroups of nonexpansive mappings were given by Brézis-Browder [4] and Hirano-Takahashi [6].

In this paper, we prove a nonlinear ergodic theorem for an amenable semigroup of nonexpansive mappings of $C$ into itself. Furthermore we obtain a necessary and sufficient condition for a left amenable semigroup of nonexpansive mappings to have a common fixed point. This is a generalization of Pazy's results [7] and [9].

2. Nonlinear ergodic theorem. Let $S$ be an abstract semigroup and $m(S)$ the Banach space of all bounded real valued functions on $S$ with the supremum norm. For each $s \in S$ and $f \in m(S)$, we define elements $f_{s}$ and $f^{s}$ in $m(S)$ given by

Received by the editors December 31, 1979 and, in revised form, April 17, 1980.

AMS (MOS) subject classifications (1970). Primary 47A35, 47H10.

Key words and phrases. Ergodic theorem, amenable semigroup, nonexpansive mapping, fixed point. 
$f_{s}(t)=f(s t)$ and $f^{s}(t)=f(t s)$ for all $t \in S$. An element $\mu \in m(S)^{*}$ (the dual space of $m(S)$ ) is called a mean on $S$ if $\|\mu\|=\mu(1)=1$. A mean $\mu$ is called left (right) invariant if $\mu\left(f_{s}\right)=\mu(f)\left(\mu\left(f^{s}\right)=\mu(f)\right)$ for all $f \in m(S)$ and $s \in S$. An invariant mean is a left and right invariant mean. A semigroup which has a left (right) invariant mean is called left (right) amenable. A semigroup which has an invariant mean is called amenable. Day [5] proved that a commutative semigroup is amenable. We also know that $\mu \in m(S)^{*}$ is a mean on $S$ if and only if

$$
\inf \{f(s): s \in S\} \leqslant \mu(f) \leqslant \sup \{f(s): s \in S\}
$$

for every $f \in m(S)$.

Now we prove a nonlinear ergodic theorem for noncommutative semigroups of nonexpansive mappings in a Hilbert space. The proof employs the methods of [8], [11] and [12].

TheOREM 1. Let $C$ be a nonempty closed convex subset of a real Hilbert space $H$ and $S$ be an amenable semigroup of nonexpansive mappings $t$ of $C$ into itself. Suppose that

$$
F(S)=\bigcap\{F(t): t \in S\} \neq \varnothing .
$$

Then, there exists a nonexpansive retraction $P$ of $C$ onto $F(S)$ such that $P t=t P=P$ for every $t \in S$ and $P x \in \overline{\operatorname{co}}\{t x: t \in S\}$ for every $x \in C$, where $\overline{c o} A$ is the closure of the convex hull of $A$.

Proof. Let $\mu$ be an invariant mean on $S$ and $x \in C$. Then, since $F(S) \neq \varnothing,\{t x$ : $t \in S\}$ is bounded and hence, for each $y$ in $H$, the real-valued function $t \rightarrow\langle t x, y\rangle$ is in $m(S)$. Denote by $\mu_{t}\langle t x, y\rangle$ the value of $\mu$ at this function. By linearity of $\mu$ and of the inner product, this is linear in $y$; moreover, since

$$
\left|\mu_{t}\langle t x, y\rangle\right| \leqslant\|\mu\| \cdot \sup _{t}|\langle t x, y\rangle| \leqslant\left(\sup _{t}\|t x\|\right) \cdot\|y\|,
$$

it is continuous in $y$, so by the Riesz theorem, there exists an $x_{0} \in H$ such that $\mu_{t}\langle t x, y\rangle=\left\langle x_{0}, y\right\rangle$ for every $y \in H$. Setting $P x=x_{0}$, we have

$$
P x \in \overline{c o}\{t x: t \in S\} \text {. }
$$

In fact, if $P x \notin \overline{c o}\{t x: t \in S\}$, then by the separation theorem there exists a $y_{0} \in H$ such that

$$
\left\langle P x, y_{0}\right\rangle\left\langle\inf \left\{\left\langle z, y_{0}\right\rangle: z \in \overline{\operatorname{co}}\{t x: t \in S\}\right\} .\right.
$$

So, we have

$$
\begin{aligned}
\inf _{t}\left\langle t x, y_{0}\right\rangle & \leqslant \mu_{t}\left\langle t x, y_{0}\right\rangle=\left\langle P x, y_{0}\right\rangle \\
& \left\langle\inf \left\{\left\langle z, y_{0}\right\rangle: z \in \overline{\operatorname{co}}\{t x: t \in S\}\right\}\right. \\
& \leqslant \inf _{t}\left\langle t x, y_{0}\right\rangle .
\end{aligned}
$$

This is a contradiction. Let $s \in S$. Then we have

$$
\begin{aligned}
0 \leqslant & \left\|t x-x_{0}\right\|^{2}-\left\|s t x-s x_{0}\right\|^{2} \\
\leqslant & \left\|t x-s x_{0}\right\|^{2}+2\left\langle t x-s x_{0}, s x_{0}-x_{0}\right\rangle \\
& +\left\|s x_{0}-x_{0}\right\|^{2}-\left\|s t x-s x_{0}\right\|^{2}
\end{aligned}
$$


and hence

$$
\begin{aligned}
0 \leqslant & \mu_{t}\left(\left\|t x-s x_{0}\right\|^{2}+2\left\langle t x-s x_{0}, s x_{0}-x_{0}\right\rangle+\left\|s x_{0}-x_{0}\right\|^{2}-\left\|s t x-s x_{0}\right\|^{2}\right) \\
= & \mu_{t}\left\|t x-s x_{0}\right\|^{2}+2\left\langle x_{0}-s x_{0}, s x_{0}-x_{0}\right\rangle \\
& +\left\|s x_{0}-x_{0}\right\|^{2}-\mu_{t}\left\|t x-s x_{0}\right\|^{2} \\
= & 2\left\langle x_{0}-s x_{0}, s x_{0}-x_{0}\right\rangle+\left\|s x_{0}-x_{0}\right\|^{2} \\
= & -\left\|x_{0}-s x_{0}\right\|^{2} .
\end{aligned}
$$

This implies $s x_{0}=x_{0}$ for every $s \in S$ and hence we have $s P x=P x$ for every $s \in S$. From

$$
\langle P s x, y\rangle=\mu_{t}\langle t s x, y\rangle=\mu_{t}\langle t x, y\rangle=\langle P x, y\rangle
$$

and

$$
\left\langle P^{2} x, y\right\rangle=\mu_{t}\langle t P x, y\rangle=\mu_{t}\langle P x, y\rangle=\langle P x, y\rangle,
$$

it follows that $P s=P$ for every $s \in S$ and $P^{2}=P$. At last, we prove that $P$ is nonexpansive. In fact, we have

$$
\begin{aligned}
\|P x-P y\|^{2} & =\langle P x-P y, P x-P y\rangle=\mu_{t}\langle t x-t y, P x-P y\rangle \\
& \leqslant\left(\sup _{t}\|t x-t y\|\right) \cdot\|P x-P y\| \\
& \leqslant\|x-y\| \cdot\|P x-P y\|
\end{aligned}
$$

for every $x, y \in C$.

As a direct consequence, we have

Corollary 1. Let $C$ be a nonempty closed convex subset of a real Hilbert space $H$ and $S$ be a commutative semigroup of nonexpansive mappings $t$ of $C$ into itself. Suppose that $F(S) \neq \varnothing$. Then there exists a nonexpansive retraction $P$ of $C$ onto $F(S)$ such that $P t=t P=P$ for every $t \in S$ and $P x \in \overline{c o}\{t x: t \in S\}$ for every $x \in C$.

By the method of Theorem 1, we can prove the following

THEOREM 2. Let $C$ be a nonempty closed convex subset of a real Hilbert space $H$ and $S$ be a left amenable semigroup of nonexpansive mappings $t$ of $C$ into itself. Then, $F(S) \neq \varnothing$ if and only if there exists an $x_{0} \in C$ such that $\left\{t x_{0}: t \in S\right\}$ is bounded.

As direct consequences, we obtain Pazy's results [7] and [9].

COROLlaRY 2. Let $C$ be a nonempty closed convex subset of a real Hilbert space $H$ and $T$ be a nonexpansive mapping of $C$ into itself. Then, $F(T) \neq \varnothing$ if and only if there exists an element $x_{0} \in C$ such that the sequence $\left\{T^{n} x_{0}: n=1,2, \ldots\right\}$ is bounded.

COROllary 3. Let $C$ be a nonempty closed convex subset of a real Hilbert space $H$ and $S=\{S(t): t \geqslant 0\}$ be a nonexpansive semigroup on $C$. Then, $F(S) \neq \varnothing$ if and only if there exists an element $x_{0} \in C$ such that $\left\{S(t) x_{0}: t \geqslant 0\right\}$ is bounded. 


\section{REFERENCES}

1. J. B. Baillon, Un théorème de type ergodique pour les contractions non linéaires dans un espace de Hilbert, C. R. Acad. Sci. Paris Sér. A-B 280 (1975), 1511 - 1514.

2. Q Quelques propriétés de convergence asymptotique pour les semigroupes de contractions impaires, C. R. Acad. Sci. Paris Sér. A-B 283 (1976), 75-78.

3. J. B. Baillon and $\mathrm{H}$. Brézis, Une remarque sur le comportement asymptotique des semigroupes non linéaires, Houston J. Math. 2 (1976), 5-7.

4. H. Brézis and F. E. Browder, Remarks on nonlinear ergodic theory, Adv. in Math. 25 (1977), 165-177.

5. M. M. Day, Amenable semigroups, Illinois J. Math. 1 (1957), 509-544.

6. N. Hirano and W. Takahashi, Nonlinear ergodic theorems for nonexpansive mappings in Hilbert spaces, Kōdai Math. J. 2 (1979), 11-25.

7. A. Pazy, Asymptotic behavior of contractions in Hilbert space, Israel J. Math. 9 (1971), 235-240.

8. _ On the asymptotic behavior of iterates of nonexpansive mappings, Israel J. Math. 26 (1977), 197-204.

9. On the asymptotic behavior of semigroups of nonlinear contractions in Hilbert space, J. Funct. Anal. 3 (1978), 292-307.

10. W. Takahashi, Fixed point theorem for amenable semigroup of nonexpansive mappings, Kōdai Math. Sem. Rep. 21 (1969), 383-386.

11. Invariant functions for amenable semigroups of positive contractions on $L^{1}$, Kōdai Math. Sem. Rep. 23 (1971), 131-143.

12. Ergodic theorems for amenable semigroups of positive contractions on $L^{\mathbf{1}}$, Sci. Rep. Yokohama Nat. Univ. Sect. I 19 (1972), 5-11.

Department of Information Sciences, Tokyo Institute of Technology, OH-OKayama, MeguroKU, TOKYO, JAPAN 